\title{
Hidrogenación de cinamaldehído sobre \\ catalizadores Au/ZrO2 y Au/ZrO2-SiO2. \\ Efecto del soporte y método de preparación
}

\author{
Cinnamaldehyde hydrogenation over \\ $\mathrm{Au} / \mathrm{ZrO} 2$ and $\mathrm{Au} / \mathrm{ZrO2}-\mathrm{SiO} 2$ catalysts. \\ Support effect and preparation method
}

\author{
Hugo A. Rojas*, José J. Martínez*, Lizbeth Vargas*, Maira I. Alvarado*, \\ Gloria Borda* $§$ \\ * Grupo de Catálisis, Escuela de Ciencias Químicas, Universidad Pedagógica y Tecnológica de \\ Colombia UPTC, Avenida Central del Norte, Vía Paipa, Tunja, Boyacá-Colombia. \\ §hugo.rojas@uptc.edu.co,jsjbnny@hotmail.com,yenny.vargas@uptc.edu.comairai88@hotmail. \\ comgborda98@hotmail.com
}

(Recibido: Diciembre 08 de 2011 - Aceptado: Noviembre 19 de 2012)

\begin{abstract}
Resumen
Se prepararon catalizadores de oro soportados en $\mathrm{ZrO}_{2}$ and $\mathrm{ZrO}_{2}-\mathrm{SiO}_{2}$ por impregnación incipiente (Imp.I.) y depositación-precipitación con urea (DPU) con una carga nominal del metal del $1 \%$. Los sólidos fueron caracterizados por difracción de rayos X (DRX), fisisorción de nitrógeno y reducción a temperatura programada (TPR). La actividad catalítica fue evaluada en la hidrogenación de cinamaldehído. La selectividad hacia el producto deseado es influenciada por la fase cristalina o amorfa del soporte y el método de deposición del oro.
\end{abstract}

Palabras clave: Alcohol insaturado, Amorfo, Cinamaldehído, Cristalino, Zirconia

\begin{abstract}
Gold catalysts supported on $\mathrm{ZrO}_{2}$ and $\mathrm{ZrO}_{2}-\mathrm{SiO}_{2}$ were prepared by incipient impregnation and depositionprecipitation with urea (DPU) with $1 \%$ of nominal gold loading. The solids were characterized by X-ray diffraction analysis (XRD), $\mathrm{N}_{2}$-physisorption and temperature programmed reduction (TPR). Catalytic activities were investigated in cinnamaldehyde hydrogenation. The selectivity towards the desired product was influenced by the crystalline or amorphous phase support and method of gold deposition.
\end{abstract}

Keywords: Amorphous, Cinnamaldehyde, Crystalline, Unsaturated alcohol, $\mathrm{ZrO}_{2}$. 


\section{Introducción}

El cinamaldehído (3-fenil-2-propenal) es un aldehído $\alpha-\beta$ insaturado que está presente naturalmente en la corteza de los árboles del cinamomo y otras especies del género Cinnamomum, como el alcanfor y la canela, ampliamente usados como saborizantes $\mathrm{y}$ aromatizantes. Por otra parte, el cinamaldehído representa la materia prima para la síntesis de importantes productos en la industria química. De la hidrogenación del cinamaldehído (CALD), el aldehído saturado (hidroxicinamaldehído (HCALD)) y el alcohol insaturado (alcohol cinamílico (COL)), se pueden obtener mediante la hidrogenación selectiva del enlace conjugado $\mathrm{C}=\mathrm{C}$ y del enlace $\mathrm{C}=\mathrm{O}$ respectivamente. Estos productos se emplean en gran medida para la preparación de productos farmacéuticos y derivados usados para la producción de perfumes (Li et al. 1997).

Se ha demostrado que el $\mathrm{Au}$ puede ser usado para reacciones de hidrogenación selectivas (Mohr et al. 2000; Claus et al. 2004) y factores como el método de preparación y soporte empleado, (Milone et al. 2006) influyen en el tamaño de partícula y por tanto podrían alterar la actividad catalítica. El método de preparación de catalizadores de Au más común es la depositación-precipitación (DP) de oro en soportes de óxidos metálicos anfóteros. Métodos eficientes como la depósitaciónprecipitación con urea (DPU) permite obtener pequeñas partículas entre 2 y $3 \mathrm{~nm}$, evitando la pérdida del oro en solución, (Haruta 2002) y además, dado que todo el oro precipita en la superficie del soporte, es posible tener un control del porcentaje final del oro sobre el mismo. Los métodos de impregnación aunque se usan comúnmente para la preparación de otros catalizadores metálicos soportados, ya que son simples y fáciles de escalar, no permiten obtener nanopartículas de oro debido a que no se elimina los cloruros residuales, los cuales, han estado asociados a la aglomeración de las partículas de oro durante la calcinación, así como a un efecto de envenenamiento de cloruros sobre los sitios activos del catalizador (Hugon et al. 2008).

Por otra parte, el soporte, es esencial para dispersar la fase activa, pero en el caso de catalizadores de $\mathrm{Au}$ las interacciones metalsoporte determinan el comportamiento catalítico en muchas de las reacciones donde este se emplea, así dependiendo de la fase presente del soporte se determina el estado de oxidación que adquiera el $\mathrm{Au}$ (Bond et al. 2006). Este aspecto es esencial para la hidrogenación de aldehídos $\alpha, \beta$-insaturados, dado que se requiere centros deficientes de electrones y sitios ácidos de Lewis en la superficie del catalizador para promover la formación del alcohol insaturado debido a que proporciona sitios adecuados de adsorción para el grupo carbonilo $\mathrm{C}=\mathrm{O}$, (Mäki-Arvela et al. 2005). Un aumento en la densidad electrónica de los centros activos del metal disminuye la probabilidad de activación del enlace $\mathrm{C}=\mathrm{C}$, por consiguiente disminuye la selectividad en la hidrogenación de enlaces olefínicos. Por otro lado, además del doble enlace $\mathrm{C}=\mathrm{C}$ y el grupo carbonilo, la molécula de cinamaldehído, contiene un grupo fenilo que puede tener una considerable influencia estérica, sobre los rendimientos de los diferentes productos de reacción.

En este trabajo, se compara el método de preparación de depósitación-precipitación con urea (DPU) e impregnación incipiente (Imp.I.) de catalizadores de $\mathrm{Au}$ soportados en $\mathrm{ZrO}_{2}$ y $\mathrm{ZrO}_{2} / \mathrm{SiO}_{2}$, modificando el método de impregnación con la formación previa de $\mathrm{Au}(\mathrm{OH})_{3}$, además de someter estos sólidos a un proceso de reducción con hidrógeno en lugar de la calcinación convencional (Baatz et al. 2008). Estos catalizadores fueron evaluados en la reducción quimioselectiva del grupo $\mathrm{C}=\mathrm{O}$ del cinamaldehído para la obtención del alcohol cinamílico y se determinó la influencia de la cristalinidad del soporte con el propósito de evaluar su posible incidencia en la actividad y selectividad obtenida.

\section{Metodología}

\subsection{Síntesis de los soportes}

El óxido se zirconio fue preparado por el método descrito por Clearfield, (Baatz et al. 2008). Una disolución de $0,1 \mathrm{M}$ de oxinitrato de zirconilo $\mathrm{ZrO}\left(\mathrm{NO}_{3}\right)_{2}$ y $0.05 \mathrm{M}$ de $\mathrm{NH}_{4} \mathrm{OH}$ se 
sometió a reflujo suavemente por $65 \mathrm{~h}$ al final se ajustó el $\mathrm{pH}$ a 10 con $\mathrm{NH} 4 \mathrm{OH}$ concentrado (15.8M). El precipitado se filtró, con posteriores lavados y se sometió a secado a $383 \mathrm{~K}$ por 12 h. El óxido mixto de $\mathrm{ZrO}_{2} / \mathrm{SiO}_{2}$ fue sintetizado por impregnación de $\mathrm{ZrO}\left(\mathrm{NO}_{3}\right)_{2}$ sobre sílice en medio acuoso, posterior secado a $393 \mathrm{~K}$. Ambos sólidos fueron calcinados en flujo de aire (50 $\mathrm{ml} / \mathrm{min}$ ) a $773 \mathrm{~K}$ por $4 \mathrm{~h}$.

\subsection{Síntesis de los catalizadores}

Los catalizadores fueron sintetizados por el método de depósitación-precipitación con urea e impregnación incipiente (Imp.I). Para el método de DPU se adicionó a los soportes una solución acuosa del precursor metálico al 1\% de $\mathrm{HAuCl}_{4}$ con urea en concentración 100 veces más alta que la concentración del precursor. Dicha suspensión se mantuvo en calentamiento a $363 \mathrm{~K}$ agitando vigorosamente durante $3 \mathrm{~h}$. Posteriormente, la muestra se separó mediante centrifugación, lavando al mismo tiempo con agua desionizada, se secó al vacío a temperatura ambiente y se calcinó a $673 \mathrm{~K}$ con una rampa de temperatura de $278 \mathrm{~K}$ y flujo de aire de 50 $\mathrm{ml} / \mathrm{min}$.

Para el método de impregnación incipiente (Imp.I.), se preparó una solución de $\mathrm{HAuCl}_{4} \mathrm{al}$ $1 \%$ y $\mathrm{Na}_{2} \mathrm{CO}_{3}$ mezcladas en relación molar 1:2 y se impregnaron con el volumen de poro de los sólidos, bajo fuerte agitación a temperatura ambiente, los sólidos se secaron bajo vacío. El catalizador se redujo en atmosfera de $\mathrm{H}_{2}$ en $\mathrm{Ar}$ al $10 \%$.

\subsection{Caracterización de los catalizadores}

Se realizaron análisis de difracción de rayos $\mathrm{X}$ (DRX) para determinar las fases cristalinas de los soportes y los catalizadores en un equipo $X$ 'Pert + PRO system PANalytical (configuración: Bragg-Brentano). Las propiedades texturales de los sólidos se evaluaron por fisisorción de nitrógeno a $77 \mathrm{~K}$ en un equipo Micromeritics ASAP 2020. Los análisis de reducción a temperatura programada (TPR) para determinar la reducibilidad del metal, se efectuaron en un equipo Autochem 2920.

\subsection{Evaluación catalítica}

Las reacciones catalíticas de hidrogenación del cinamaldehído, en fase líquida se realizaron en un reactor tipo batch a $363 \mathrm{~K}$, a una presión de $\mathrm{H}_{2}$ de 0,63 MPa, utilizando 0,2 gramos de catalizador y una solución de cinamaldehído 0,1 M utilizando n-heptano como solvente. El seguimiento de la reacción se realizó durante 7 $\mathrm{h}$, previo a la reacción, los catalizadores fueron reducidos a $573 \mathrm{~K}$ en corriente de $\mathrm{H}_{2}$ con un flujo de $50 \mathrm{ml} / \mathrm{min}$. Para el seguimiento de los productos de reacción, se utilizó un cromatógrafo de gases Varian 3800 y los productos se identificaron mediante un espectrómetro de masas Saturn 2000.

\section{Resultados y discusión}

\subsection{Caracterización de los sólidos}

Con el fin de determinar las especies componentes de los catalizadores, se llevó a cabo un estudio de difracción de rayos $\mathrm{X}$ y así identificar las especies cristalinas de los soportes. La figura 1 muestra el patrón de difracción del soporte $\mathrm{ZrO}_{2}$ y $\mathrm{ZrO}_{2} / \mathrm{SiO}_{2}$, las reflexiones de $\mathrm{ZrO}_{2}$ son características de la fase monoclínica correspondiente al grupo espacial [P121/C1] tipo Baddeleyita. En el difractograma de rayos $\mathrm{X}$ de la muestra $\mathrm{ZrO} / \mathrm{SiO}_{2}$ aparece un pico amorfo a $20^{\circ}$ indicativo de la sílice, los demás picos en 25,49 y $52^{\circ}$ son indicativos de zirconia tetragonal con buena cristalinidad, así es posible señalar que pequeños agregados de zirconia se hayan depositado sobre la sílice de manera no uniforme.

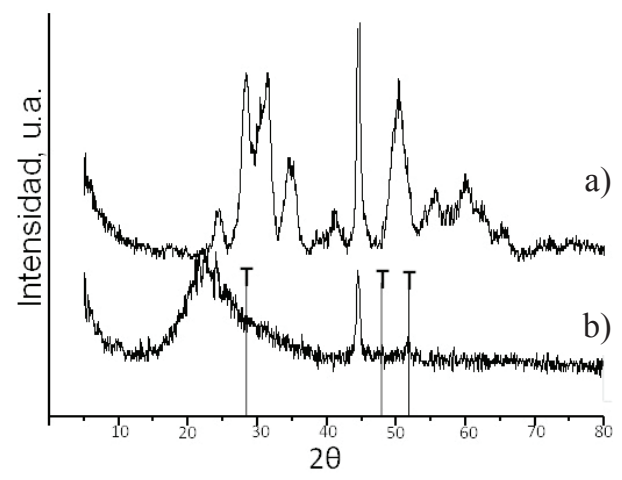

Figura 1: Difractogramas de los cristales sintetizados: a) Zirconia cristalina, b) Zirconia amorfa. 
En los patrones de difracción de rayos X para los catalizadores de $\mathrm{Au}$ (figura 2), las reflexiones típicas del metal se encuentran muy cercanas a las del soporte; en ninguno de los casos se observó un aumento de intensidad de las reflexiones próximas del $\mathrm{Au}$, comportamiento que se debe a la sensibilidad de la técnica.

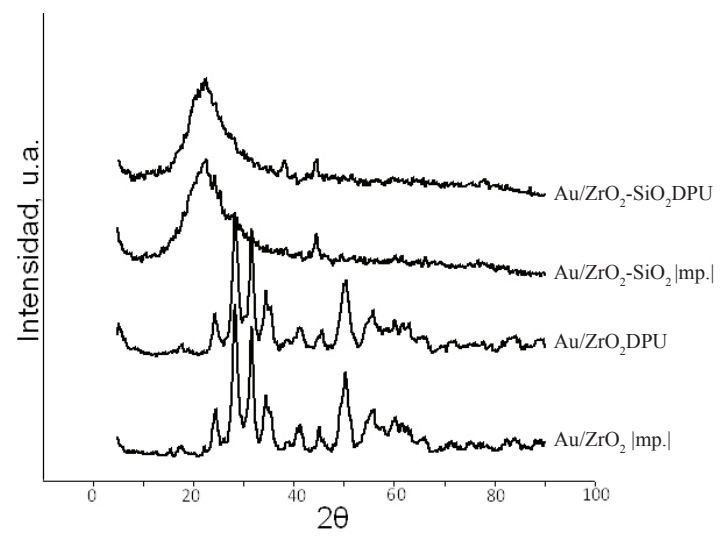

Figura 2: $\mathrm{DRX}$ de $\mathrm{Au} / \mathrm{ZrO}_{2}$ y $\mathrm{Au} / \mathrm{ZrO}_{2}-\mathrm{SiO}_{2}$ sintetizados por los métodos DPU e Imp.I.

Para el estudio de las propiedades texturales de los catalizadores se determinaron isotermas de $\mathrm{N}_{2}$ a $77 \mathrm{~K}$. Los resultados obtenidos se resumen en la figura 3 y en la tabla 1. Las isotermas de nitrógeno a $77 \mathrm{~K}$ (Figura 3), permitieron calcular el área superficial específica de los sólidos $\left(\mathrm{ZrO}_{2}\right.$ y $\left.\mathrm{ZrO}_{2}-\mathrm{SiO}_{2}\right)$, en la figura 3 se evidencia que las muestras presentaron isotermas del tipo IV, características de sólidos mesoporosos (Gregg et al. 1982). Como puede observarse las isotermas obtenidas en cada uno de los sólidos indican distinta porosidad y cristalinidad de los soportes, así, aunque ambas son de tipo IV, la curva de histéresis para $\mathrm{ZrO}_{2}$ monoclínica, es de tipo $\mathrm{H} 4$, propio de materiales con poros tubulares o de cuello de botella, haciendo difícil determinar la rama de desorción, mientras que en $\mathrm{ZrO}_{2} \mathrm{SiO}_{2}$ se exhibe una histéresis de tipo H1 la cual está asociada a la presencia de poros cilíndricos que presentan un ciclo angosto con sus ramas de adsorción y desorción paralelas entre sí.

En la tabla 1, se evidencian las propiedades texturales tanto de los sólidos como de los catalizadores, puede observarse, para la zirconia en fase monoclínica y amorfa que estos sólidos presentan una elevada área superficial, resultado interesante, si se tiene en cuenta que el óxido de zirconio comercial, presenta un área superficial cercana a los $50 \mathrm{~m}^{2} / \mathrm{g}$ (Chuah \& Jaenicke 1997).

Por otra parte, la incorporación de Au no afecta significativamente las propiedades texturales del soporte, como se muestra en la tabla 1 . Sin embargo, la disminución en el volumen de poro se asocia a un bloqueo parcial de poros del soporte por cristales de oro.

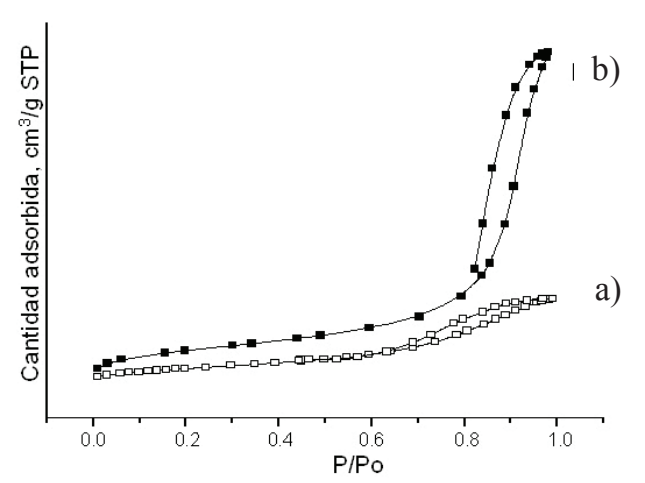

Figura 3. Isotermas de adsorción de los soportes. a) $\mathrm{ZrO}_{2}$ y b) $\mathrm{ZrO}_{2}-\mathrm{SiO}_{2}$

Tabla 1. Características de los catalizadores

\begin{tabular}{ccccc}
\hline Catalizador & Método & $\mathrm{S}_{\mathrm{BET}}, \mathrm{m}^{2} \mathrm{~g}^{-1}$ & $\mathrm{~V}_{\mathrm{p}}, \mathrm{mlg}^{-1}$ & $\mathrm{~S}_{\mathrm{p}}, \mathrm{nm}$ \\
\hline $\mathrm{ZrO}_{2}$ & Reflujo & 137 & 0,24 & 6,97 \\
$\mathrm{ZrO}_{2}-\mathrm{SiO}_{2}$ & Imp. & 253 & 0,82 & 13,01 \\
$\mathrm{Au} / \mathrm{ZrO}_{2}$ & Imp.I. & 135 & 0,26 & 6,95 \\
$\mathrm{Au} / \mathrm{ZrO}_{2}$ & DPU & 134 & 0,25 & 6,83 \\
$\mathrm{Au} / \mathrm{ZrO}_{2}-\mathrm{SiO}_{2}$ & Imp.I. & 250 & 0,84 & 12,97 \\
$\mathrm{Au} / \mathrm{ZrO}_{2}-\mathrm{SiO}_{2}$ & DPU & 251 & 0,79 & 12,91 \\
\hline
\end{tabular}

Mediante ensayos de reducción a temperatura programada se obtuvieron los perfiles redox de los catalizadores preparados por los métodos de DPU e Imp.I. (Figura 4). Los perfiles de TPR de los catalizadores de oro reportados en la bibliografía dependen en gran medida del soporte utilizado (Venkov et al. 2006; Idakiev et al. 2006). En la figura 4, se observa que aparece un primer pico de reducción a $350 \mathrm{~K}$, excepto para $\mathrm{Au} / \mathrm{ZrO}_{2}-\mathrm{SiO}_{2}$ preparado por DPU. Un segundo pico aparece cerca de los $400 \mathrm{~K}$, sin embargo se observa un desplazamiento hacia temperaturas superiores, que puede atribuirse al tipo de soporte y el método de preparación, una explicación a tal desplazamiento se relaciona con la formación de distintas especies de oro; 
se considera además, que con el empleo de $\mathrm{ZrO}_{2}-\mathrm{SiO}_{2}$, es posible que existan agregados de $\mathrm{ZrO}_{2}$ sobre la superficie de la sílice $\left(\mathrm{SiO}_{2}\right)$, de esta forma el metal puede depositarse indistintamente ya sea sobre la superficie de $\mathrm{ZrO}_{2}$ o $\mathrm{SiO}_{2}$, que ocasiona el desplazamiento de los picos. Cuando el Au se deposita por DPU los picos de reducción pueden explicarse por la reducción de los distintos complejos $[\mathrm{Au}(\mathrm{OH}) \mathrm{n}$ $\mathrm{Cl}_{4-\mathrm{n}}$ ]- depositados sobre el soporte, (Hutchings 2008) debido a que no es posible eliminar por completo los residuos clorados provenientes del precursos metálico. Los picos observados a mayores temperaturas en $\mathrm{Au} / \mathrm{ZrO}_{2}$ DPU y Au/ $\mathrm{ZrO}_{2}-\mathrm{SiO}_{2} \mathrm{DPU}$ pueden estar asociados a otros procesos de reducción presentes en la superficie, probablemente por la posible reducción de especies de $[\mathrm{AuCl}(\mathrm{OH}) \mathrm{x}]$-, este proceso sería similar al que se presenta con catalizadores de $\mathrm{Pt} / \mathrm{TiO}_{2}$, donde los iones cloruro coordinados en el complejo $\mathrm{PtO}_{\mathrm{x}} \mathrm{Cl}_{\mathrm{y}}$ inhiben la reducción del metal y pueden ser removidas solo hasta los $673 \mathrm{~K}$ (Hwang C-P et al., 1996). Las especies formadas en Imp.I se reducen a mayor temperatura que en DPU. Por otra parte, la diferencia de temperatura en la reducción de $\mathrm{Au}$ en $\mathrm{ZrO}_{2}-\mathrm{SiO}_{2}$, se asigna a la interacción del soporte con el oro.

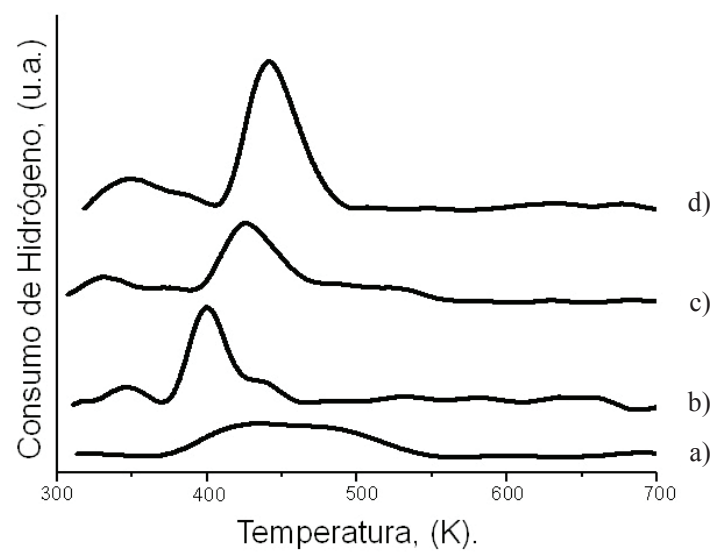

Figura 4. Perfiles de reducción a temperatura programada de los catalizadores a) $\mathrm{Au} / \mathrm{ZrO}_{2}-\mathrm{SiO}_{2}-\mathrm{DPU}$. b) $\mathrm{Au} / \mathrm{ZrO}_{2}-$ DPU. c) $\mathrm{Au} / \mathrm{ZrO}_{2}-\mathrm{SiO}_{2}$ Imp. I. d) $\mathrm{Au}-\mathrm{ZrO} \mathrm{O}_{2}$ Imp.I.

\subsection{Actividad catalítica}

La tabla 2 resume la actividad catalítica a 3 horas de reacción y selectividad a una isoconversión del 5\%. Puede observarse que la conversión depende del soporte empleado y no del método de preparación. Los valores similares indican que la deposición del oro es distinta en cada soporte, la baja actividad en el óxido mixto estaría asociada a un mayor tamaño de partícula. De acuerdo con los resultados de Bus et al., (Bus et al., 2005) cúmulos de oro con un diámetro inferior a $2 \mathrm{~nm}$. son esenciales para obtener una alta actividad y selectividad en la hidrogenación de cinamaldehído.

Tabla 2. Actividad catalítica a tres horas de reacción.

\begin{tabular}{cccccc}
\hline Catalizador & Método & Conv. $3 \mathrm{~h}$ & $\mathrm{~S}_{\mathrm{C}=\mathrm{C}}$ & $\mathrm{S}_{\mathrm{C}=\mathrm{O}}$ & $\mathrm{S}_{\text {Otros }}$ \\
\hline $\mathrm{Au} / \mathrm{ZrO}_{2}$ & Imp.I. & 13 & 13 & 68 & 19 \\
$\mathrm{Au} / \mathrm{ZrO}_{2}$ & DPU & 13 & 54 & 41 & 5 \\
$\mathrm{Au} / \mathrm{ZrO}_{2}-\mathrm{SiO}_{2}$ & Imp.I. & 4 & 50 & 17 & 33 \\
$\mathrm{Au} / \mathrm{ZrO}_{2}-\mathrm{SiO}_{2}$ & DPU & 4 & 41 & 0 & 59 \\
\hline
\end{tabular}

Los resultados de selectividad muestran para el óxido mixto una menor selectividad hacia el alcohol insaturado. Similares resultados han sido observados con el uso de coloides de Au$\mathrm{PVA} / \mathrm{ZrO}_{2}$ (PVA: polivinilalcohol) donde se observa una $\mathrm{S}_{\mathrm{C}=\mathrm{O}}$ cercana al $30 \%$, mientras que con Au-PVA/SiO $/ \mathrm{Si}_{2}$ no se evidencia la formación del alcohol cinamílico, (Shi et al. 2008), tal como en el caso de $\mathrm{Au} / \mathrm{ZrO}_{2}-\mathrm{SiO}_{2}$ DPU. Puede verse claramente que el soporte mixto no favorece la actividad catalítica en la hidrogenación de cinamaldehído independiente del método de preparación empleado.

\section{Conclusiones}

Los resultados de DRX indicaron la presencia de especies cristalinas presentes en los catalizadores de $\mathrm{Au} / \mathrm{ZrO}_{2}$ en fase monoclínica y $\mathrm{Au} / \mathrm{ZrO}_{2}-\mathrm{SiO}_{2}$ en donde la zirconia se presenta en fase tetragonal sobre sílice amorfa.

Los análisis de fisisorción de nitrógeno indicaron elevadas áreas superficiales en los soportes, los cuales no mostraron modificación en sus propiedades texturales con la incorporación del Au por ninguno de los métodos de anclaje del metal.

Se presenta mejor comportamiento catalítico tanto en actividad como en términos de 
selectividad en los catalizadores de $\mathrm{Au}$ preparados por ambos métodos (DPU y Imp.I) soportados en Zirconia monoclinica

\section{Agradecimientos}

$\mathrm{Se}$ agradece el soporte financiero COLCIENCIAS-SENA-UPTC bajo el proyecto $\mathrm{N}^{\mathrm{o}} 110948925094$.

\section{Referencias bibliográficas}

Baatz, C., Decker, N., \& Prüße, U. (2008). New innovative gold catalysts prepared by an improved incipient wetness method. Journal of Catalysis, 258 (1), 165-169.

Bond, G. C., Louis, C., \& Thompson, D.T. (2006) Catalysis by gold. London: Imperial College Press.

Bus, E., Miller, J. T., \& Van Bokhoven, J. A. (2005). Hydrogen chemisorption on $\mathrm{Al}_{2} \mathrm{O}_{3}$ supported gold catalysts. Journal of Physical Chemistry B 109 (30), 14581-14587.

Chuah, G. K., \& Jaenicke, S. (1997). The preparation of high surface area zirconiaInfluence of precipitating agent and digestion. Applied Catalysis A: General 163 (1-2), 261273.

Claus, P., Hofmeister, H., \& Mohr, C. (2004). Identification of active sites and influence of real structure of gold catalysts in the selective hydrogenation of acrolein to allyl alcohol. Gold Bulletin 37 (3-4), 181-186.

Gregg S. J. (1982). Adsorption, Surface Area and Porosity. Ber. Buns. Chem 86, 957-957.

Haruta, M. (2002). Catalysis of gold nanoparticles deposited on metal oxides. Cattech 6 (3), 102-115

Hwang, C-P, \& Yeah, C-T. (1996) Platiunmoxide species formed bu oxidation of platinum crystallites supported on alumina. Journal of Molecular Catalysis A: Chemical 112 (2), 127138
Hugon, A., Delannoy, L., \& Louis, C. (2008). Supported gold catalysts for selective hydrogenation of 1,3-butadiene in the presence of an excess of alkenes. Gold Bulletin 41 (2), $127-138$

Hutchings, G. J. (2008). Supported gold and gold palladium catalysts for selective chemical synthesis. Catalysis Today 138 (1-2), 9-14

Idakiev, V., Tabakova, T., Naydenov, A., Yuan, Z. Y., \& Su, B. L. (2006). Gold catalysts supported on mesoporous zirconia for low-temperature water-gas shift reaction. Applied Catalysis B: Environmental 63 (3-4), 178-186

Li, G. J., Li, T., Xu, Y. D., Wong, S. T., \& Guo, X. X. (1997). Selective hydrogenation of cinnamaldehyde to cinnamyl alcohol on L-zeolite supported catalysts. Studies in Surface Science and Catalysis105B, 1203.

Mäki-Arvela, P., Hájek, J., Salmi, T., \& Murzin, D. Y. (2005). Chemoselective hydrogenation of carbonyl compounds over heterogeneous catalysts. Applied Catalysis A: General 292 (12), 1-49.

Milone, C., Ingoglia, R., \& Galvagno, S. (2006). Gold supported on iron oxy-hydroxides: A versatile Tool for the Synthesis of Fine Chemicals. Gold Bulletin 39 (2), 54-65.

Mohr, C., Hofmeister, H., Lucas, M., \& Claus, P. (2000). Gold catalysts for the partial hydrogenation of acrolein. Chemical Engineering and Technology 23 (4), 324-328.

Shi, H., Xu, N., Zhao, D., \& Xu, B. Q. (2008). Immobilized PVA-stabilized gold nanoparticles on silica show an unusual selectivity in the hydrogenation of cinnamaldehyde. Catalysis Communications 9 (10), 1949-1954.

Venkov, T., Klimev, H, Centeno, M. A., Odriozola, J. A., \& Hadjiivanov, K. (2006). State of gold on an $\mathrm{Au} / \mathrm{Al}_{2} \mathrm{O}_{3}$ catalyst subjected to different pre-treatments: An FTIR study. Catalysis Communications 7 (5), 308-313. 